\title{
Politique commerciale de diversification et Diversification internationale des exportations de la Côte d'Ivoire
}

\author{
Dr Oumar DJIBRILA \\ Université Jean Lorougnon GUEDE, UFR SEG, Côte d'Ivoire, Daloa
}

\begin{abstract}
Résumé: La croissance économique des pays d'Afrique subsaharienne après leur indépendance s'est fondée sur les exportations des matières premières, surtout d'origine agricole, en vertu de la théorie de l'avantage absolu et comparatif et surtout de l'existence d'un marché mondial. En vue d'analyser ou de mesurer l'impact de la diversification internationale sur la croissance économique ivoirienne, nous procéderons à une analyse de sa politique commerciale avant 1980 . Ensuite, nous examinons, l'effet de sa politique commerciale sur ses échanges commerciaux de 1980 à 2012, date importante dans l'histoire de l'évolution de la Côte d'ivoire. Le recours aux instruments de mesure caractéristiques de l'indice de Theil nous permet de montrer que la politique de diversification menée par la Côte $d^{\prime}$ Ivoire a un effet relativement moyen sur la croissance de ses exportations. Ce résultat suggère la mise en place d'une politique soutenue de transformation structurelle de l'économie ivoirienne.
\end{abstract}

Mots clés : Politique commerciale, diversification internationale, Exportation, croissance, indice de Theil.

\section{Introduction}

La Côte d'Ivoire est un pays en développement qui enregistre de bonnes perspectives de croissance économique actuelle (6,2\% selon la BM) en dépit de la Covid-19. Après la crise post-électorale de 2012, une période particulière dans l'histoire de la Cote d'Ivoire, la politique économique mis en place par le gouvernement a permis de relancer sa croissance économique (Le taux de croissance du PIB était de 4,7\%, en 2011, 9\% en 2012 et 7, 9\%, en 2014 selon la Banque Mondiale). Cette hausse de l'activité économique est aussi imputable au commerce international des produits de base issus surtout du secteur agricole qui constitue $49 \%$ de ses exportations vers ses partenaires mondiaux (CNUCED, 2014) grâce à certains produits agricoles comme le cacao, le caoutchouc, le coton, etc. De plus en plus, le pays exporte quelques produits manufacturés qui constitue $16 \%$ de ses exportations. Les exportations ivoiriennes sont surtout dominées par les produits agricoles. En dépit de la volonté de l'Etat d'industrialiser son économie, la transformation industrielle, tant voulue, reste faible.

Ayant choisi le libéralisme économique, le Côte d'Ivoire s'est résolument ouverte sur l'extérieur. Depuis les théories du commerce international, des études montrent que l'ouverture extérieure est 
un gain pour la croissance économique (CAPEC, 2014). Le commerce international favorise alors la croissance économique à travers et surtout la diversification des exportations (Berthélemy, 2005). Cet auteur démontre "le rôle majeur que peuvent jouer, dans les processus de diversification économique, les nouvelles formes du commerce international que sont les échanges intra-branche et la décomposition internationale des processus productifs " grâce à la diversification internationale du travail (DIT). Si pour Wacziarg et Imbs (2003), la diversification est le résultat mécanique d'un processus de diversification, Berthélemy (op.cit) ajoute qu'elle est « la conséquence de stratégies efficaces de participation à la mondialisation » à travers lesquelles, les pays peuvent profiter davantage de leurs avantages comparatifs dans les échanges commerciaux mondiaux tout en améliorant leurs tissus industriels. La diversification peut être entendue comme une nouvelle forme de spécialisation qui peut s'appuyer par conséquent sur le commerce intra-branche et la décomposition internationale des processus productifs. Etant, membre de l'OMC, une politique de diversification internationale réussie de la Côte d'Ivoire serait un atout pour jouir du système commercial multilatéral (SMC) soutenu par cette organisation. Les études sur la diversification, jusqu'ici se sont focalisées sur I'utilisation des indicateurs de mesures. Mais, peu d'études mettent l'accent sur la politique commerciale consistant à mettre en place une institution de promotion des exportations. La Côte d'Ivoire, depuis 2000, s'est doté d'une association pour la promotion des exportations (APEX $\mathrm{Cl}$ ) après la signature des Accords de commerciaux depuis 1995, date de création de l'OMC. L'APEXCI, qui assiste le secteur des exportations grâce à ses partenaires, a connu la fin de l'exécution de la plupart de ses projets d'appui fin 2014.

Comment la politique commerciale de diversification a-t-elle affectée la diversification des exportations en Côte d'Ivoire au cours de cette phase d'expansion économique ? A l'aide de l'indice de Theil, l'objectif est d'analyser l'effet de la politique de diversification sur la diversification des exportations de la Côte d'Ivoire. En d'autres termes, il s'agit de voir si les exportations ivoiriennes sont extensives en termes de lignes de produits nouveaux ou intensifs en termes d'accroissements de ses exportations traditionnelles. L'hypothèse de l'étude est la suivante : la politique de promotion des exportations a favorisé la diversification des exportations internationales de la Côte d'Ivoire. L'étude s'appuie donc sur la décomposition de l'indicateur de Theil sur la période 1995-2012, avant la mise en place de l'initiative pays pauvre très endetté. Les données statistiques ont été obtenues à l'aide des sites : UNComtrade, Unctadstat, Wits.

Notre travail comporte trois phases. La première partie traite de la politique de diversification commerciale. La seconde partie présente la politique de diversification en Côte et enfin, la dernière partie, empirique, analyse la diversification dans un contexte de durabilité des exportations ivoiriennes. 


\section{POURQUOI LA POLITIQUE DE DIVERSIFICATION COMMERCIALE ?}

Cette section fait ressortir l'intérêt économique de la politique de diversification, tout en mettant l'accent sur ses déterminants et ses effets économiques.

\subsection{Politique économique et Politique de la diversification économique}

Cette sous-section définit les notions de politique commerciale à la lumière de la politique économique et de diversifications horizontale et verticale.

\subsubsection{Politique économique et politique commerciale}

Selon le dictionnaire économique (Guerrien), la politique économique est un ensemble d'instruments qu'on manipule afin d'atteindre un certain nombre d'objectifs. Parmi ces instruments de politique structurelle, on distingue la politique commerciale. Elle peut être destinée soit au marché national, soit au marché international. On parle alors de politique commerciale nationale et de politique commerciale internationale.

\subsubsection{Diversification horizontale/verticale}

La diversification horizontale vise de nouveaux secteurs d'activités alors que la diversification verticale vise un élargissement de la gamme de produits dans un même secteur (Berezin, 2002). Une économie est diversifiée lorsque sa structure productive est dispersée en un grand nombre d'activités différentes les unes comparativement aux autres par la nature des biens et services fournis par la production de ses acteurs. Cette diversification peut s'appréhender à travers un indicateur synthétique et des variables économiques.

\subsection{Déterminants de la diversification}

La revue de la littérature fait ressortir les analyses aux plans théorique et empirique de la diversification. Des exemples pratiques de diversification de quelques pays sont soulignés.

\subsubsection{Fondements théoriques}

Un certain nombre de variables déterminent la diversification (CEA, 2006). Elles peuvent être regroupées en variables ou facteurs économique, politique, commerciale, etc.

\section{- Ouverture commerciale}

Il existe une relation positive entre ouverture et diversification (Melitz, 2003). Plus un pays est ouvert sur l'extérieur, plus il accroit ses « changes commerçiaux en situation de concurrence monopolistique.

\section{- Facteurs structurels}

Le travail étant un facteur de la croissance, l'accroissement du capital humain favorise la croissance des exportations d'un pays en termes de produits plus élaborés (Melitz, op. cit). 


\section{- Variables économiques}

Au plan économique, un environnement économique instable provoqué par une forte inflation n'est pas favorable à la création et au développement de nouveau secteur d'activité (Banque de France, 2007). Selon Melitz (op. cit), l'amélioration des termes de l'échange augmente la profitabilité et cela doit favoriser une hausse de la diversification.

\section{- Variables institutionnelles}

Les investissements publics peuvent contribuer à améliorer la diversification. Toutefois, des études ont mis en avant des relations non linéaires (Hammouda, Karinji et Jallab, 2009). Enfin, les politiques industrielles, les infrastructures, la gouvernance et l'aide publique peuvent contribuer à une plus grande diversification et sophistication des processus productifs si les politiques suivies sont appropriées.

\section{- Ressources naturelles}

A ces éléments, il faut ajouter les ressources naturelles. 80\%Dans les années 1960, des exportations sz des pays en développement étaient des produits de base, mais de nos jours, $80 \%$ de biens produits et exportés sont des produits industriels. Toutefois, certains pays sont tributaires de ressources naturelles en provenance du secteur minier ou des hydrocarbures (Gelb, 2010).

\subsubsection{Etudes empiriques}

A partir du calcul de l'indice d'Herfindahl, Hammouda et al. (2009) explique les déterminants de la diversification en Afrique du Nord. Dogruel et Teckce (2011) qui s'intéressent spécifiquement à la relation entre libéralisation commerciale et diversification dans les pays au Sud et à l'Est de la Méditerranée ainsi que Gourdon (2010) et Hausman et al. (2010) qui, dans un rapport pour la Banque Mondiale, proposent des analyses respectivement du cas Algérien et du rôle des politiques industrielles dans les PAN (Pays de l'Afrique du Nrd). Lim et Saborowski (2012) présentent quant à eux une analyse spécifique au cas syrien et au rôle de l'État.

Deux autres mesures de la diversification, à savoir le ratio d'exportations industrielles sur la population, permettant d'apprécier le degré de sophistication des exportations, et la décomposition de la croissance des exportations en marges extensive et intensive, qui permet de mettre en lumière les formes différenciées que prennent ces processus (Cottet et al. (2012), analysent la diversification dans les pays africains de la zone franc (PAZF), en intégrant la dimension temporelle. C'est ce qui semble échapper aux travaux de Wacziarg (2003) et de (Klinger et Lederman, 2006 ; Hesse, 2009 ; Cadot et al, 2011) pour qui la diversification va de pair avec le revenu par habitant. 


\subsection{Impacts de la Politique de diversification internationale}

La diversification économique est d'un enjeu capital depuis les années 1930, pour les politiques régionales et les politiques commerciales et occupe une place importante dans les politiques de développement (Barthélémy 2005). Elle joue un rôle important dans la croissance d'une économie grâce à l'amélioration de la productivité, aux investissements et à la stabilisation des recettes d'exportation. Son importance est même au-delà de la spécialisation et est prônée par la nouvelle théorie du commerce international.

\subsubsection{Limite de la spécialisation et diversification économique des exportations}

La nature des produits exportés par les économies subsahariennes permettent de dire qu'elles se spécialisent dans la production des exportations des matières premières d'origine agricole en vertu de la théorie des avantages comparatifs de David Ricardo. Par ailleurs, une forte concentration dans la production de biens primaires a comme conséquence de rendre ces économies vulnérables, donc sujettes aux différents aléas conjoncturels. La spécialisation qu'ont connue les économies africaines, héritée de l'économie coloniale, a connu ses limites. Lederman et Maloney (2007) montre qu'entre 1980 et 2005, le PIB/h progresse bien lentement chez les exportateurs nets de ressources naturelles $(0,66 \%)$ que chez les importateurs nets de ressources naturelles $(2,2 \%)$.

Prebich et Singer (1950) ont souligné le risque d'une concentration excessive sur les exportations des produits primaires sur la croissance et la stabilité. La question de la diversification ne doit pas se restreindre à une migration du secteur agricole vers le secteur industriel qui toutefois aide à limiter la détérioration des termes de l'échange sur les revenus des échanges commerciaux. Selon Bertinelli et al. (2006) et Levchenko et al. (2008), la diversification économique est de nos jours perçu comme un moyen de stabilisation des recettes à long terme en face d'une hausse de l'élasticité de la demande et de la volatilité des prix.

Une analyse fondée sur les théories anciennes et nouvelles du commerce international et des liens entre diversification et spécialisation fait ressortir que la spécialisation n'est pas nécessairement un obstacle à une spécialisation commerciale efficace, si cette diversification peut être associée à des facteurs qui sont identiques à ceux qui déterminent l'échange intra-branche.

\subsubsection{Théorie du commerce international et enjeu de la diversification internationale}

Selon la « nouvelle théorie du commerce international » les flux d'échange intra-branche s'appuient sur la différentiation horizontale ou la diversification verticale.

La diversification a des effets bénéfiques sur la croissance à condition que les rendements d'échelle soient croissants (Berthlémy, 2005). Cependant la diversification peut être contre-productive lorsque 
les ressources sont réallouées aux nouveaux secteurs au détriment des autres secteurs performants (Berezin, 2002).

Le succès des pays émergents est en partie imputable à leur avancée sur la voie de la diversification. Cela se corrobore par une étude menée de Berthelemy sur la Chine (2005). Il démontre, par ailleurs, que la Chine est devenue le pays qui diversifie plus et en outre résiste aux fortes fluctuations mondiales en dépit de son fort degré d'ouverture. Cependant des études comme celle de Massell (1970) montrent que les pays à économie peu diversifiée connaissent une forte instabilité due à leurs recettes d'exportation. Cette instabilité des exportations est négativement reliée à la croissance dans les pays exportateurs de ressources pétrolières (Gaskari et al. ,2011).

Si la théorie montre que la diversification favorise l'expansion du commerce international, c'est parce que cela favorise la croissance et le développement, si à partir du modèle de Romer (1990), une diversification dans les intrants est bénéfique, alors cette analyse peut s'étendre à la diversification de la production qui agit favorablement sur la croissance (Feenstra et al, 1999). Pour lui, si l'économie peut produire des biens différents et plus, toutes choses égales par ailleurs, son revenu est élevé. Mais si la production est concentrée dans un seul secteur d'activité en situation de rendement d'échelle observé, cela est un obstacle pour la réalisation de gains de productivités particulièrement dans une petite économie dole la Côte d'Ivoire.

\section{Analyse de la politique de diversification de la Côte d'ivoire}

La conception d'une bonne politique commerciale est un exercice d'équilibrage consistant à fournir des incitations aux entreprises pour qu'elles innovent, à renforcer les capacités, les investissements dans les R\&D, et modernisent la technologie (CEA, 2015). Le tableau ci-dessous indique les mesures de politique commerciale et leurs effets sur les chaines de valeurs mondiales. Cependant, la politique commerciale doit être sélective, mais protégeant les industries naissantes de la concurrence internationale pour éviter la désindustrialisation de l'économie puisque celles-ci sont dominées par les industries étrangères ayant des ressources plus importantes. C'est pourquoi, la politique sélective doit envisager un bon apport de la valeur ajoutée et l'accent devrait alors être mis sur la protection effective.

\subsection{Politiques publiques et mesures commerciales avant 1995}

\subsubsection{Intervention publique en matière de promotion des exportations}

Rappelons que la Côte d'Ivoire s'est inscrite dans le libéralisme économique. A cause de l'existence d'un marché international de matières, elle a encouragé les exportations de ses matières premières, 
notamment, le café et le cacao. Avec la crise économique des années 1980, elle a connu le programme de l'Ajustement structurel et le processus de Libéralisation du commerce extérieur a connu les phases suivantes : entre 1984 et 1986, une avancée sensible, entre 1987 et 1989, un recul des exportations, une relance progressive et sélective entre 1990 et 1993 et une accélération à la faveur de la dévaluation de 1994 (OMC).

\subsubsection{Mesures de politique commerciale}

Entre 1984 et 1986, la Côte d'Ivoire abolit un grand nombre de restrictions quantitatives à l'importation (taux de protection effectifs autour de $40 \%$ ) et introduit un système de subvention des exportations non-traditionnelles.

En 1987, les réformes sur le commerce extérieur de 1985 sont remises en cause. Les autorités publiques font face en effet à une crise économique de grande ampleur mais se placent dans une position d'attente d'une remontée des cours et de refus de l'ajustement, avec le soutien implicite de la France (Azam, 1994). La période 1990-1993 voit la reprise du processus de libéralisation du commerce, dans le cadre du PASCO (Programme d'ajustement structurel du commerce) consacré à la compétitivité et financé par la Banque Mondiale. Les diminutions de droits de porte sont progressives et sélectives (Contamin, 1999) : homogénéisation et baisse de tarifs à partir de 1990, démantèlement progressif des barrières non tarifaires à partir de 1992, élimination des licences et de la plupart des taxes à l'exportation en 1991. Le tableau ci-dessous indique les mesures commerciales sélectives et leurs effets potentiels.

Tableau 1: Mesures commerciales sélectives

\begin{tabular}{|c|c|}
\hline Mesures commerciales & Effets potentiels liés à l'investissement \\
\hline $\begin{array}{l}\text { Droits d'importation, progressivité des droits. } \\
\text { Obstacles non tarifaires: normes réglementaires } \\
\text { (par exemple obstacles techniques au commerce et } \\
\text { mesures sanitaires et phytosanitaires). }\end{array}$ & $\begin{array}{l}\text { Effet négatif sur les investissements dans le secteur } \\
\text { des exportations dans les } \\
\text { opérations faisant intervenir la teneur en } \\
\text { importations faisant l'objet de la mesure. } \\
\text { Effet positif sur les investissements à la recherche de } \\
\text { marchés ou en vue de la } \\
\text { substitution de produits locaux aux importations } \\
\text { (saut d'obstacle). }\end{array}$ \\
\hline $\begin{array}{l}\text { Préférences pour le développement de l'accès } \\
\text { aux marchés (par exemple Système généralisé } \\
\text { de préférences, initiative « Tout sauf les } \\
\text { armes », loi sur la croissance et les possibilités } \\
\text { économiques de l'Afrique). }\end{array}$ & $\begin{array}{l}\text { Effet positif sur les investissements étrangers dans } \\
\text { les pays bénéficiant de préférences } \\
\text { ciblant les exportations destinées aux pays donneurs } \\
\text { de préférences. }\end{array}$ \\
\hline
\end{tabular}

Source : Conférence des Nations Unies sur le commerce et le développement (2013). 


\subsection{Politiques publiques et mesures commerciales après 1995}

En adhérant à l'OMC, en 1995, la Cl a souscrit ainsi à l'engagement unique. Ainsi, elle est membre du système commercial multilatéral (SCM). Elle respecte donc ses engagements en matière de droits de douanes et impositions. L'OMC permet les accords de partenariat et en prélude à la fin des accords de Cotonou en 2007, I'UE a initié des négociations sur les APE qui doivent remplacer ces derniers accords. En 2013, le gouvernement ivoirien a ratifié un « APE intérimaire » qui permet à la Côte d'ivoire de conserver ses avantages à l'accès préférentiel aux marchés européens (ITC, 2014).

\subsubsection{Mesures de politique commerciale}

Le tableau ci-dessous résume les politiques commerciales adoptées par la Côte d'Ivoire en tant que partie prenante des Accords de l'OMC de 1995.

\section{Tableau 1: Mesures commerciales sélectives}

\begin{tabular}{|c|c|}
\hline $\begin{array}{l}\text { Accords préférentiels ou } \\
\text { accords de libre échange } \\
\text { (notamment les règles d'origine } \\
\text { et les accords sectoriels). }\end{array}$ & $\begin{array}{l}\text { Effet positif sur les investissements qui bénéficient d'un commerce plus } \\
\text { facile (et moins cher) entre les pays membres, renforcement des chaînes } \\
\text { de valeur régionales. } \\
\text { Effet positif sur les investissements à la recherche de marchés par le biais } \\
\text { d'économies d'échelle résultant de l'activité au service d'un marché plus } \\
\text { grand. } \\
\text { Effet de consolidation sur les investissements (principalement grâce à des } \\
\text { fusions et à des acquisitions) par suite d'une reconfiguration des chaînes } \\
\text { de valeur mondiales dans les pays membres. }\end{array}$ \\
\hline $\begin{array}{l}\text { Facilitation du commerce } \\
\text { (s'appliquant tant aux } \\
\text { Importations qu'aux } \\
\text { exportations). } \\
\text { Promotion des exportations (par } \\
\text { exemple financement des } \\
\text { exportations, garanties du } \\
\text { crédit et foires commerciales). }\end{array}$ & $\begin{array}{l}\text { Effet positif sur les investissements dans le secteur des exportations par la } \\
\text { réduction du coût des passages multiples des frontières s'agissant tant des } \\
\text { importations que des exportations et par le biais d'exportations accélérées } \\
\text { (revêtant un intérêt particulier dans le domaine des chaînes de valeur } \\
\text { mondiales pour lesquelles le facteur temps est déterminant). } \\
\text { Effet positif sur les investissements à la recherche de marchés qui } \\
\text { bénéficient d'importations facilitées (et rendues moins chères). }\end{array}$ \\
\hline $\begin{array}{lr}\text { Mesures } & \text { commerciales } \\
\text { correctives (par exemple } \\
\text { pratiques } & \text { antidumping, } \\
\text { garanties et droits } \\
\text { compensateurs). }\end{array}$ & $\begin{array}{l}\text { Effet négatif sur les investissements orientés vers les exportations dans le } \\
\text { pays touché par la mesure (et sur les investisseurs présents dans le secteur } \\
\text { des exportations qui ont pris des décisions d'investir avant l'adoption de la } \\
\text { mesure). }\end{array}$ \\
\hline
\end{tabular}

Source : Conférence des Nations Unies sur le commerce et le développement (2013). 


\subsubsection{Appui institutionnel et promotion des exportations après 1995 : Rôle et missions de I'APEXI}

L'échec des marchés a fait donc naître des agences de promotion des exportations qui méritent d'être analysées rigoureusement (Marcio, 2014). Ainsi, il souligne que plusieurs auteurs ont analysé l'impact des agences de promotion des exportations soutenues par les Etats sur les marges extensives. Une étude du même auteur a mis en exergue l'impact positif de l'agence de promotion brésilienne des exportations sur les entreprises initialement non-exportatrices de produits. Qu'en est-il de l'Association de promotion des exportateurs de la Côte d'Ivoire ?

L'Apexi (Association pour la promotion des exportations de Côte d'Ivoire) est une structure mise en place pour la promotion des exportations de la Côte d'Ivoire. Elle joue deux principaux rôles à travers plusieurs missions :

- Rôle de promotion et de développement des exportations : A ce niveau, en tant qu'agence de promotion et de développement des exportations, elle favorise la croissance soutenue des exportations de la Côte d'Ivoire à travers la réduction de la dépendance vis-à-vis des produits traditionnels; l'accroissement de la part des exportations à forte valeur ajoutée; la diversification géographique des exportations et la création d'une culture d'exportation.

Parmi ces missions, la diversification des exportations est très importante car elle a pour but d'accroitre la proportion des exportations ivoiriennes mais celle à forte valeur ajoutée. Outre la diversité des biens ivoiriens, les produits manufacturés de qualité sont indispensables à la pérennité de la diversification. Cela nécessite par conséquent un minimum d'investissements dans la technologie.

- Rôle de promotion et développement du secteur privé : Au nombre des missions à lui assignées, celles d'assurer la compétitivité des entreprises en mettant l'accent sur les PME au vue de leur apport dans le PIB et d'assurer un environnement propice au développement du secteur privé.

Par les acteurs de la croissance économiques, les PME concernent plus de $80 \%$ et occupent une place prépondérante dans l'environnement économique ivoirien. Si nous tenons compte de la mondialisation et de la concurrence internationale aussi bien au niveau des biens traditionnels de production que des produits manufacturés, ce rôle est également un atout pour la promotion des exportations et donc pour la diversification internationale.

L'association de promotion des exportations agit par le biais de certaines activités liées aux projets et aussi grâce aux opportunités qu'offrent les marchés internationaux comme l'AGOA ("African gowth 
opportunity act »). Pour atteindre ses missions, L'association bénéficie de l'appui de d'autres institutions partenaire aussi bien sur le plan régional que sur le plan international. Au niveau régional, ce sont l'Union Economique et Monétaire Ouest Africain (UEMOA) et la Communauté Economique de Développement Economique de l'Afrique de l'Ouest (CEDEAO) et au niveau international, ce sont la Banque Africaine de Développement (BAD), la Banque Mondiale (BM), l'International Trade Center (ITC), I'Organisation Mondiale du Commerce (OMC), I'Organisation des Nations Unies (ONU), I'Union européenne (UE), I'Organisation Internationale de la Francophonie (OIF) et le groupe Afrique Caraïbes Pacifiques (ACP).

Sur la base des financements de ses partenaires, l'APEX CI fait la promotion des exportations à travers différents projets qu'elle a mis en place. Ainsi, à travers le système d'information régionale des affaires de la CEDEAO, sa mission est de fournir des informations économiques, statistiques et permettre aux opérateurs de la sous-région de saisir des opportunités d'affaires avec ceux de la sous-région. En outre, I'APEX $\mathrm{Cl}$ appuie les PME sur la base du coût du renforcement des capacités des entreprises. Par ailleurs, grâce à son projet de renforcement des organisations interprofessionnelles (OI), elle accompagne les entreprises ivoiriennes en facilitant l'accès aux marchés publics de la Zone UEMOA (Union économique et monétaire ouest africain). Hayakawa (2011) et Marcio (2014) ont fait des études qui soutiennent que les agences de promotion des exportations affectent positivement les exportations.

\section{Analyse de la diversification internationale et durabilité des exportations ivoiriennes}

Cette section présente la structure des échanges commerciaux internationaux, leurs analyses et le recours aux indicateurs de mesure de la diversification afin d'apprécier la diversification internationale des exportations ivoiriennes.

\subsection{Structure et évolution géographique des exportations commerciales}

Ci-dessous, la structure comparative des exportations ivoiriennes de quelques principaux produits d'exportation.

\subsubsection{Structure des exportations commerciales ivoiriennes}

Le graphique 3.1 représente les exportations agricoles en dollar de la $\mathrm{Cl}$ vers les partenaires que I'Afrique subsaharienne et le Monde de 1995 à 2012. 
Figure 1: Exportations en valeur des produits agricoles de la $\mathrm{Cl}$

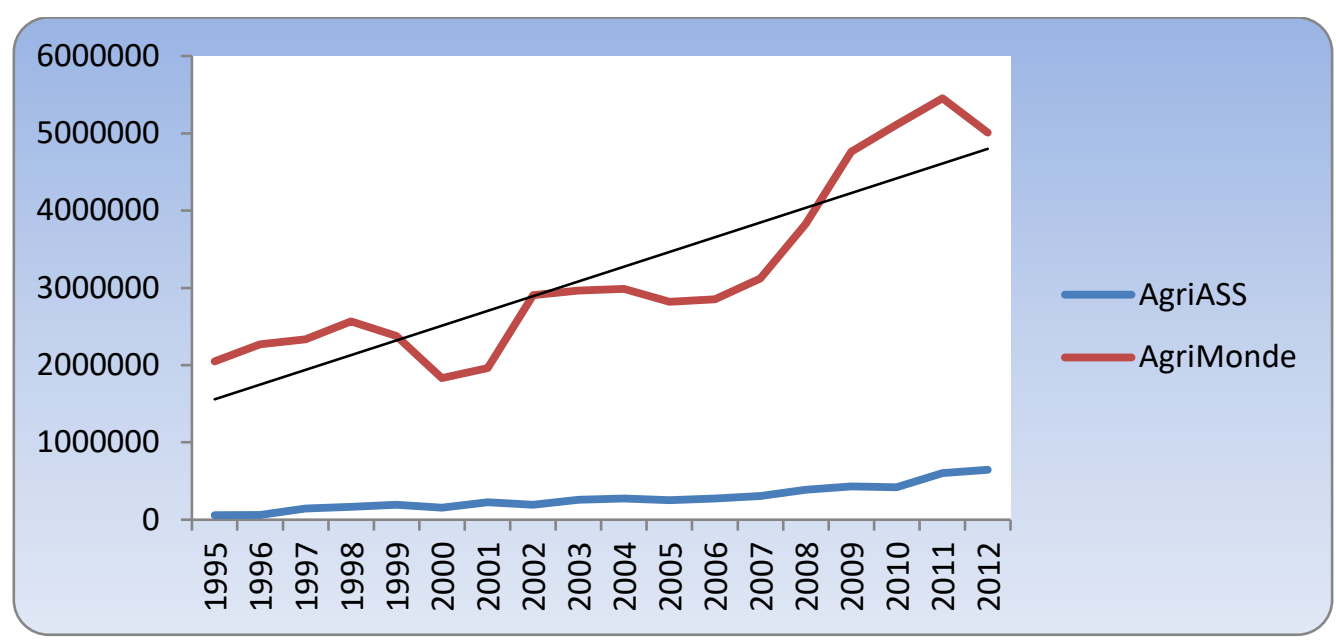

Auteur à partir des calculs sur Wits, 2014

Les courbes représentatives des exportations en valeur issues des exportations de produits agricoles indiquent que les exportations agricoles suivent une tendance globalement haussière. Cependant, les exportations mondiales sont largement au-dessus des exportations vers l'Afrique subsaharienne (ASS). Ces tendances démontrent que la Côte exporte plus ses ressources agricoles vers le Monde qui est fortement demandeur de matières premières agricoles. La libéralisation des échanges est donc plus en faveur du Monde que l'Afrique Subsaharienne (ASS). La Côte d'Ivoire, à l'image des pays de l'ASS produit plus de matières premières essentiellement agricoles destinées à la transformation dans les pays industriellement développés. Le graphique 3.2 montre l'évolution comparée des exportations de produits industriels vers le monde et l'Afrique Subsaharienne (ASS) en dollar.

Figure 2: Evolution comparée Monde/ASS des exportations industrielles de la Cl

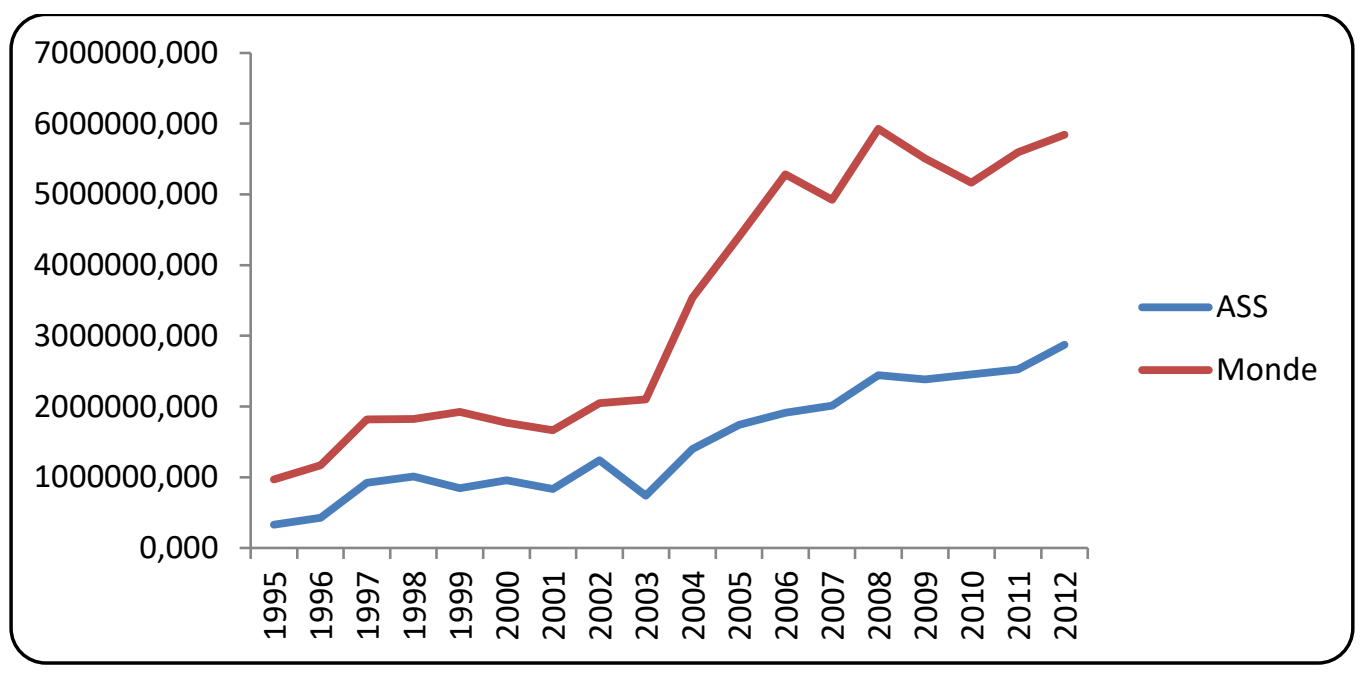

Source : Auteur à partir des données sur wits, 2014 
On remarque que les exportations vers le monde sont largement au-delà des exportations vers I'Afrique subsaharienne. La politique commerciale de la Côte d'Ivoirienne favorise davantage les exportations hors de ses groupes d'intégrations (UEMOA, CEDEAO) en matière de commerce régional. Comment évoluent les exportations ivoiriennes ?

\subsubsection{Tendance des exportations commerciales ivoiriennes}

Ci-dessous, l'évolution du commerce international de la Côte d'Ivoire en dollar.

Figure 3: Evolution en valeur du commerce international de la $\mathrm{Cl}$

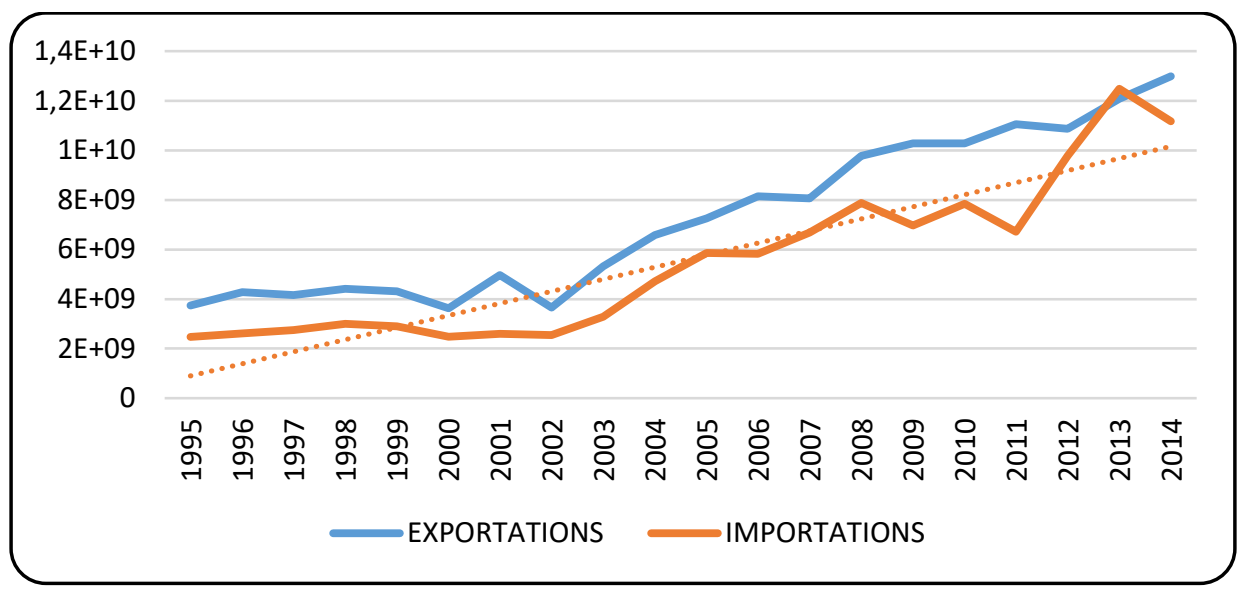

Auteur, à partir de UNcomtrade, 2015

L'évolution de la courbe traduit une évolution positive des exportations ivoiriennes (balance commerciale excédentaire) depuis son accession à l'OMC. Cela traduit la volonté des autorités ivoirienne de favoriser les exportations ivoiriennes en mettant en place une politique commerciale pour assurer la durabilité des exportations. Ci-dessous, l'évolution des exportations de la Côte d'ivoire.

Le graphique 3.1 montre l'évolution du taux d'exportations de la $\mathrm{Cl}$ vers le partenaire Monde.

Tableau 1: Evolution en pourcentage des exportations de la $\mathrm{Cl}$

\begin{tabular}{lrrrrrrrrrr}
\hline ANNEES & 1995 & $\mathbf{1 9 9 6}$ & $\mathbf{1 9 9 7}$ & $\mathbf{1 9 9 8}$ & $\mathbf{1 9 9 9}$ & $\mathbf{2 0 0 0}$ & $\mathbf{2 0 0 1}$ & $\mathbf{2 0 0 2}$ & $\mathbf{2 0 0 3}$ & $\mathbf{2 0 0 4}$ \\
\hline TAUX & - & $14 \%$ & $-3 \%$ & $6 \%$ & $-2 \%$ & $-16 \%$ & $37 \%$ & $-27 \%$ & $46 \%$ & $24 \%$ \\
\hline ANNEES & $\mathbf{2 0 0 5}$ & $\mathbf{2 0 0 6}$ & $\mathbf{2 0 0 7}$ & $\mathbf{2 0 0 8}$ & $\mathbf{2 0 0 9}$ & $\mathbf{2 0 1 0}$ & $\mathbf{2 0 1 1}$ & $\mathbf{2 0 1 2}$ & $\mathbf{2 0 1 3}$ & $\mathbf{2 0 1 4}$ \\
\hline TAUX & $10 \%$ & $12 \%$ & $-1 \%$ & $21 \%$ & $5 \%$ & $0 \%$ & $7 \%$ & $-2 \%$ & $11 \%$ & $\mathbf{7} \%$ \\
\hline
\end{tabular}

Source : Auteur à partir d'UNcomeTrade

Pour mieux analyser les exportations ivoiriennes, nous procédons à la présentation de l'évolution des taux de croissance des exportations en pourcentage par le graphique 3.4.

Figure 4: Evolution du taux de croissance des exportations de la Cl 


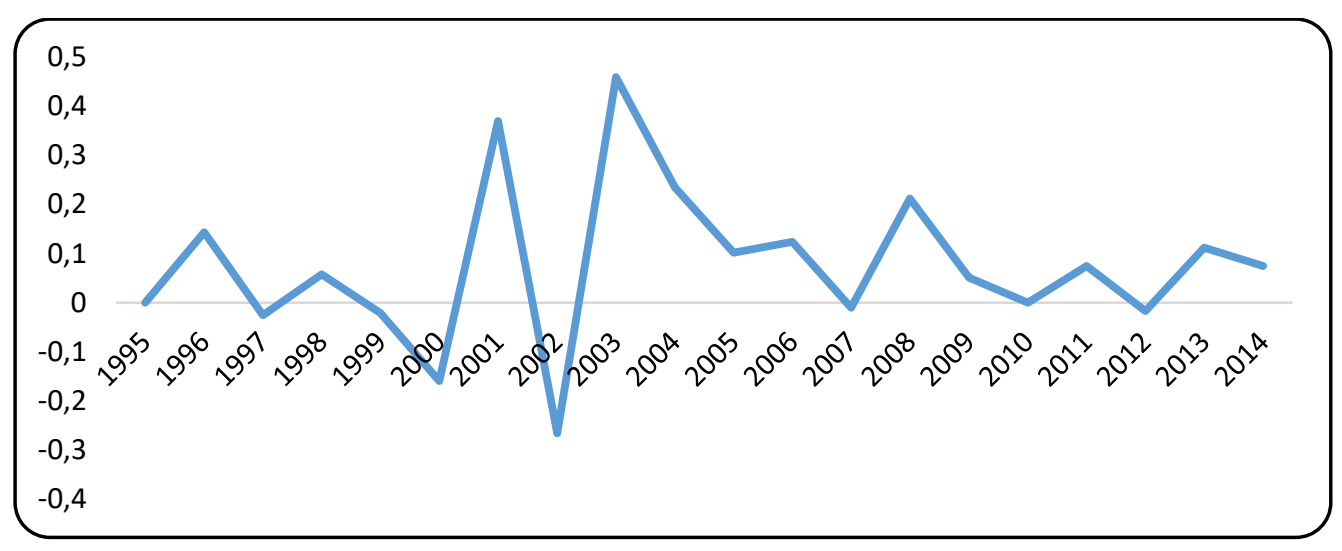

Source : auteur à partir des données statistiques sur WTO, 2015

Le taux de croissance des exportations de marchandises a une allure très erratique. L'évolution comporte des phases de croissance et décroissance. Le caractère erratique est plus prononcé de 2000 à 2003. De 1995 à 2000, nous constatons une baisse tendancielle du taux de croissance des exportations de marchandises. Ce taux de croissance se relève et s'accélère (37\%) en 2001 et est suivi d'une phase décroissante rapide entre 2001 et 2002 (Taux de croissance négatif). Et à partir de cette période jusqu'à 2003, le taux des exportations augmentent pour atteindre $46 \%$ en 2003 . Nous constatons une baisse tendancielle du taux de croissance jusqu'à 2007. De 2007 à fin 2008, nous avons relèvement du taux de croissance des exportations qui connaîtra une tendance baissière jusqu'en 2010 avec un taux de croissance pratiquement nul des exportations. En effet, l'économie ivoirienne a enregistré des contre-performances à l'issue de la crise militaro-politique de 2002. Les exportations ont connu une légère contraction au début de la crise, mais ont repris moyennement continue pour atteindre 5063 milliards de FCFA en 2010. La balance commerciale est structurellement excédentaire et s'élevait à 1181 milliards de FCFA en 2010 (OMC, 2012). A partir de 2011, le taux de croissance évolue positivement, mais pas significativement. En effet, de 2\% en 2011, il croît de 11\% en 2014.

\subsection{Diversifications horizontale et verticale}

Cette partie met présente les secteurs d'activité économique qui ont connu une diversification, ainsi que les produits issus de cette diversification à la lumière de la politique de promotion des exportations menée par l'Association de promotion des exportations de la Côte d' Ivoire (APEX-CI). Le graphique cidessous indique le top 10 des marchés d'exportation de marchandises de la $\mathrm{Cl}$ vers les 10 premiers pays partenaires en 2014, en dollar. 
Figure 5: Exportations vers les dix (10) premiers pays partenaires de la $\mathrm{Cl}$

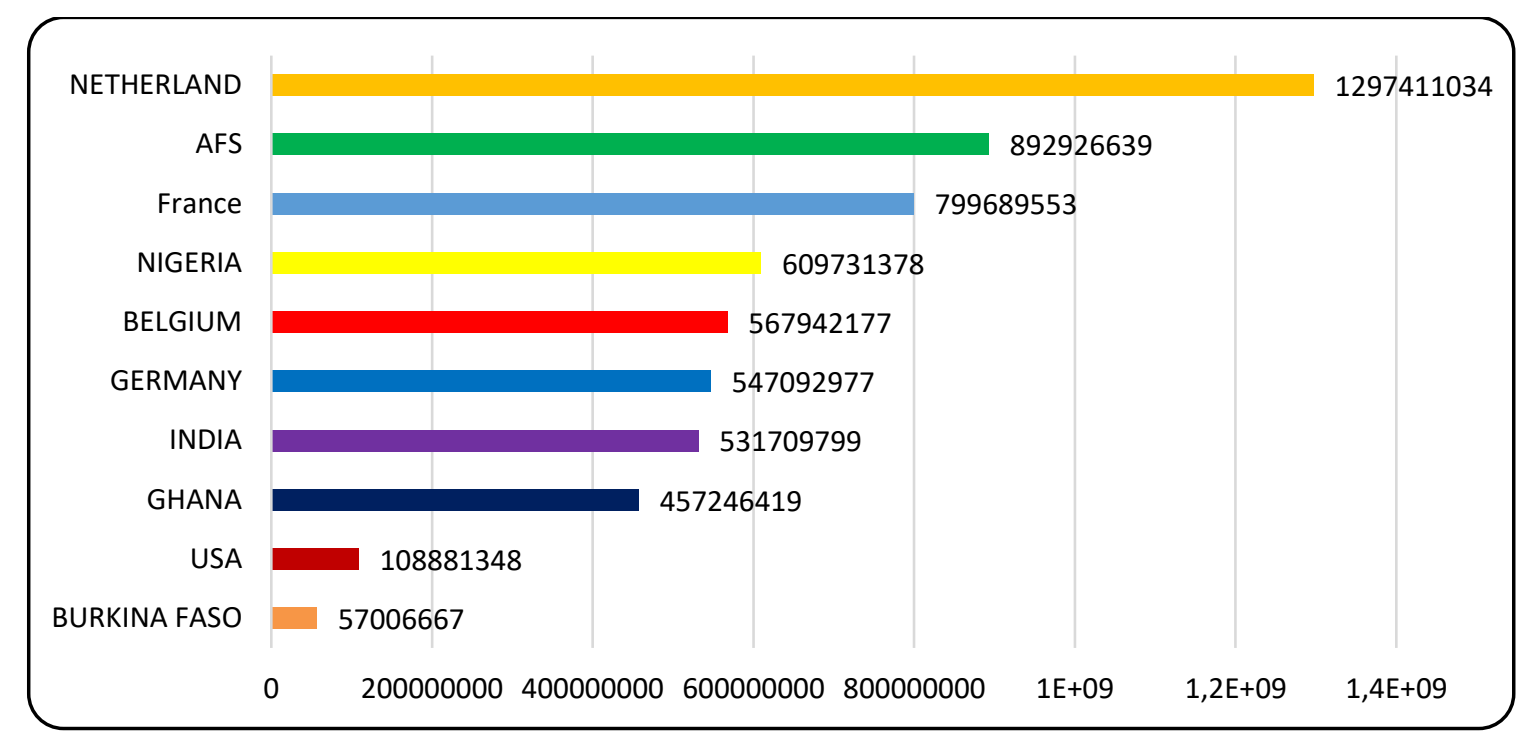

\section{Source : auteur à partir d'UNcomtrade}

Cette évolution indique que la Côte d'Ivoire diversifie géographiquement ses exportations. La France n'est plus définitivement le premier pays d'exportation de la Côte d'Ivoire. Elle exporte plus vers I'Afrique du Sud (AFS), un pays appartenant au groupe BRICS (Brésil, Russie, Inde, Chine et South Africa). Néanmoins, trois pays de la CEDEAO (Nigéria, Ghana et Burkina Faso) sont parmi les dix principaux partenaires de la Côte d'Ivoire en matière d'exportations.

\subsubsection{Secteurs d'exportation}

L'économie ivoirienne comprend les trois secteurs traditionnels que sont les secteurs primaire, secondaire et tertiaire.

Le secteur agricole est essentiellement composé des cultures pérennes comme le cacao $\left(1^{\mathrm{er}}\right.$ pays producteur mondial), le café et le coton. Le secteur manque de financement et est insuffisamment transformé. Quant au secteur secondaire, il est caractérisé par un secteur industriel dominé par les industries agroalimentaires et chimiques. Enfin, le secteur tertiaire est celui des services (36,8\% du PIB, en 2010). Le tableau ci-dessous indique le total des marchandises et des services exportés par la Côte d'ivoire (UNCTAD, 2014).

Tableau 2: Exportations des marchandises et services de la $\mathrm{Cl}$

\begin{tabular}{lcccc}
\hline En millions (\$US) & $\mathbf{2 0 0 5}$ & $\mathbf{2 0 1 0}$ & $\mathbf{2 0 1 3}$ & $\mathbf{2 0 1 4}$ \\
\hline $\begin{array}{l}\text { Exportations } \\
\text { marchandises }\end{array}$ & 7697 & 11410 & 13247 & 12783 \\
$\begin{array}{l}\text { Exportations services } \\
\text { 9 } 934\end{array}$ & 1183 & 935 & - \\
\hline
\end{tabular}

Source : Unctadstats, 2015 
L'analyse statistique de l'évolution du commerce international montre que les exportations de marchandises ont baissé de 3,5\% de 2013 à 2014. Les exportations de services ont, par contre connu une hausse de 27,6\% en 2013. Malgré cette contre-performance au niveau des exportations de biens, la balance commerciale est restée excédentaire.

\subsubsection{Structure des exportations par groupe de Produits}

En 2014, la structure des dix principales exportations ivoiriennes, en dollar, sont représentées par la figure ci-dessous.

Figure 6: Structure des produits d'exportation de la Cl

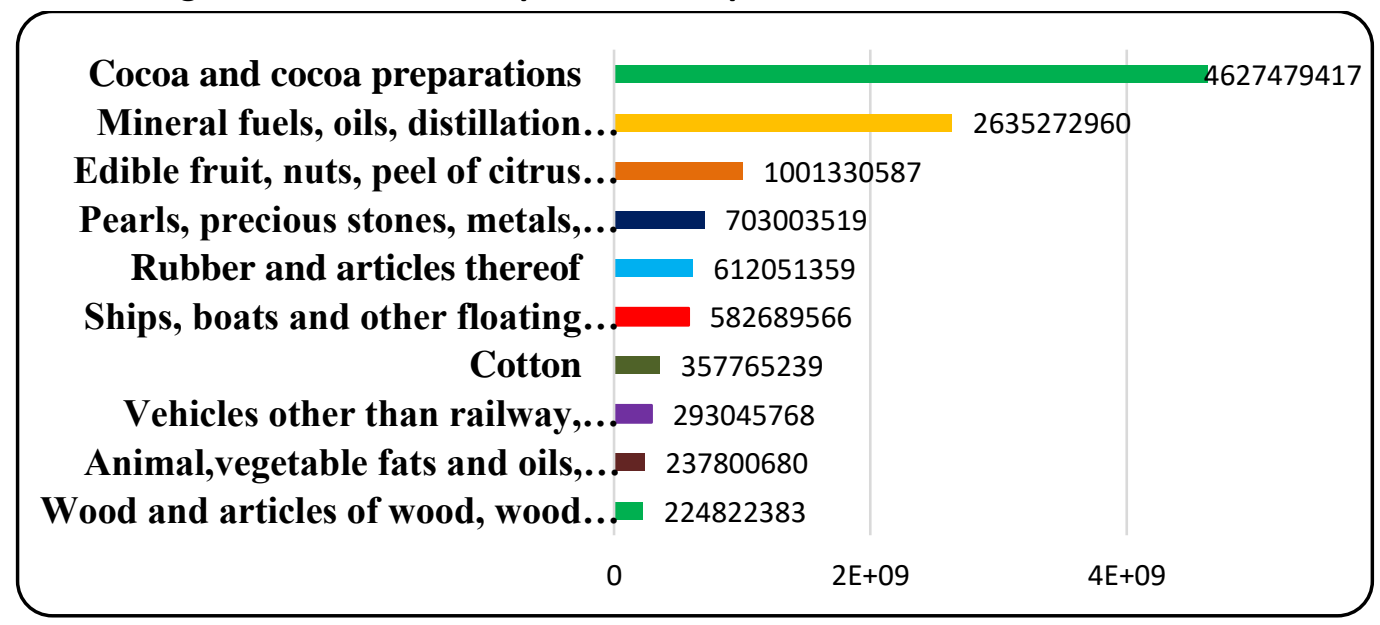

Source : auteur, à partir des données UNcomtrade, 2015

L'analyse graphique indique que les exportations ivoiriennes sont diversifiées, avec en tête toujours, la dominance des exportations de cacao. L'évolution de la structure des produits indique que la Côte d'Ivoire diversifie ses exportations. Un principal produit des exportations, le café et en outre, l'hévéa ne figurent pas dans ce classement, mais apparaissaient dans les statistiques de 2005 et 2010 (UNcomtrade).

\subsection{Indicateurs de mesures et analyse des effets de la politique de diversification}

La méthodologie d'approche se fonde sur le recours d'un indicateur composite de mesure de la diversification; afin de cerner l'impact de la politique de diversification sur la diversification internationale en termes de croissance durable des exportations ivoiriennes, voire d'accroissement des ressources d'exportation.

\subsubsection{Mesures de la diversification économique internationale}

Plusieurs indicateurs sont utilisés pour mesurer la diversification des exportations. Dans les études d'économie internationale (de Peretti et al. 2002), l'indice de concentration (Berthélémy, 2005) sert d'instruments de mesures. A partir des travaux de Melitz (2003), d'autres indicateurs ont été préférés. 
Les études de Carrère, Strauss-Kahn et Cadot (2011) basées sur des indicateurs de mesure globale ont laissé, de plus en plus, la place à une diversité de mesures désagrégées de la diversification.

Ci-dessous, l'analyse des principaux indices et leurs interprétations possibles.

\section{Indices de mesures globales}

Les indices de concentration les plus couramment utilisés sont les indices Herfindahl-Hirschmann, de Gini et de Theil (distribution des revenus). L'indice de Herfindahl-Hirschmann (HH) a été mis au point par Orris Herfindahl en 1950. Pour un pays, pris à une date donnée, l'indice HH de concentration des exportations normalisé (pour des valeurs entre 0 et 1 ) est donné par la formule suivante (CNUCED, 2013) :

$$
H=\frac{\sum_{k=1}^{n}\left(\mathrm{~s}_{k}\right)^{2}-1 / n}{1-1 / n} \text { avec } s_{k}=x_{k} / \sum_{k=1}^{n} x_{k}
$$

Où $s_{k}$ est la part des exportations de la ligne $k$, (avec $x_{k}$ la valeur exportée) dans le total des exportations et $\mathrm{n}$ le nombre de lignes d'exportations. L'indice de Herfindahl - Hirschmann $(\mathrm{HH})$ mesure la dispersion des parts dans les exportations totales des produits. Une valeur de l'indice proche de 1 correspond à une concentration très forte du marché sur le produit en question. En revanche, une valeur proche de 0 traduit une répartition plus homogène du commerce entre les exportateurs ou les importateurs. À l'origine, cette méthode permet de déterminer à quel point une industrie est oligopolistique, de même le contrôle d'un marché donné par les plus grandes entreprises industrielles (Hammouda et al. 2006).

Fourni par le secrétariat de la CNUCED (2013), cet indice HH peut servir de mesure supplémentaire de la spécialisation/diversification des exportations (Gutierrez de Pinières et Ferrantino 1997, Imbs et Wacziarg, 2003 et CESAP, 2004 ; Hammouda et al. 2006). L'indice de Gini a été largement employé sous diverses formulations équivalentes. Il peut se calculer plus simple en ordonnant d'abord les items des exportations par taille croissante et en calculant le cumul des parts d'exportations $X_{k}$. Le coefficient de Gini s'obtient par la formule la suivante :

$$
G=1-\frac{1}{n} \sum_{k=1}^{n}\left(\mathrm{X}_{k}-\mathrm{X}_{k-1}\right) \text { avec } X_{k}=\sum_{l=1}^{k} s_{l}
$$

GINI mesure l'inégalité de la distribution des données considérées, c'est-à-dire des exportations. Une valeur élevée de n'indice correspond à un niveau de concentration élevé des exportations. Enfin de compte, l'indice de Theil (1972) est également très utilisé. Sa formule est la suivante : 


$$
T=\frac{1}{n} \sum_{i=0}^{1} \frac{x_{i}}{\bar{x}} \cdot \log \left(\frac{x_{i}}{\bar{x}}\right)
$$

Où n, est le nombre total des produits exportés; $\bar{x}$ est la moyenne des flux d'exportations xi. Pour Jacquemin et Berry (1979) et Barthélémy (2005) cet indice est préférable aux deux autres, car il est décomposable en sous-groupe. Tous ces indices sont sensibles au degré de désagrégation, mais n'intègrent pas le niveau technologique et le contenu des exportations.

\section{Indices composites}

L'indice de Theil peut être décomposé selon deux concentrations : à la marge extensive et à la marge intensive. La concentration à la marge intensive se perçoit par l'inégalité des parts des exportations dans les lignes actives; la concentration à la marge extensive est associée à un nombre restreint de lignes actives (Carrère, Strauss-Kahn et Cadot, 2011). Il s'en suit une définition de la diversification aux deux marges : la diversification à la marge intensive entre deux dates $t_{0}$ et $t_{1}$ qui se traduit par une convergence des parts des biens exportations dans un panier les biens initialement exportés à la date $t_{0}$ et la diversification à la marge extensive qui est associée à une hausse du nombre des lignes actives ou lignes de produits.

La décomposition de l'indice de Theil permet de distinguer la diversification intra-groupe et la diversification intergroupe. Cette décomposition est utilisée pour mesurer la diversification à la marge intensive (intra-groupe) et la diversification à la marge extensive (intergroupe).

Soit $\mathrm{n}$, le nombre total de lignes de produits exportés qu'on divise en nombre de lignes actives et en nombre de lignes inactives. Soit $n_{j}($ pour $j=0,1)$ le nombre de lignes actives de produits exportés :

- La diversification intergroupe s'obtient par la formule suivante :

$$
T^{B}=\sum_{j=0}^{1} \frac{n_{j}}{n} \frac{\bar{x}_{j}}{\bar{x}} \cdot \log \left(\frac{\bar{x}_{j}}{\bar{x}}\right)
$$

- La diversification intragroupe est déterminée par :

$$
T^{W}=\sum_{j=0}^{1} \frac{n_{j}}{n} \frac{\bar{x}_{j}}{\bar{x}} \cdot T^{j}=\sum_{j=0}^{1} \frac{n_{j}}{n} \frac{\bar{x}_{j}}{\bar{x}}\left[\frac{1}{n} \sum_{k \in j}^{1} \frac{x_{j}}{\bar{x}_{j}} \cdot \log \left(\frac{x_{j}}{\bar{x}_{j}}\right)\right]
$$

Tj est la valeur de l'indice de Theil pour les sous-groupes j=0,1. Il est facile de vérifier que la somme de la variabilité intragroupe $T^{\mathrm{W}}$ et la variabilité intergroupe $\mathrm{T}^{\mathrm{B}}$ est égale à la variabilité totale de l'indice globale de Theil : $\mathrm{T}=\mathrm{T}^{\mathrm{B}}+\mathrm{T}^{\mathrm{W}}$. Avec $\bar{x}$ est la valeur moyenne des exportations en dollars, $\bar{x}$ j est la valeur 
moyenne des exportations du groupe $\mathrm{j}$, et $x_{i}$ est la valeur des exportations du produit i dans le groupe j.

\section{Justification du recours à l'indice de Theil}

La décomposition de l'indice de Theil est très importante, car la diversification à la marge extensive et à la marge intensive reflètent l'évolution réelle des activités productives d'un pays. Elle peut donner un premier aperçu de l'orientation des politiques de diversification plus accrue aux deux marges, qui devraient découler normalement de recommandations différentes (Carrère, Strauss-Kahn et Cadot, 2011).

\section{Analyse de la diversification par l'indice de Theil}

Parmi ces outils d'analyse de la diversification, cet indice quoique son interprétation semble compliquée, ses composantes ne prennent pas en compte ni, le niveau technologique, ni, le contenu des exportations. Ces indicateurs nous permettront de comprendre les sources de blocage du processus de diversification.

La diversification suppose la création de nouvelles lignes de produits d'exportation d'une part et d'autre part, l'accroissement ou le maintien des produits d'exportation déjà dans la base des produits exportés (Cadot, 2009 ; Besedes et Prusa, 2011).

\subsubsection{Impacts de la diversification économique sur le commerce international}

\section{Indice de Theil global}

A l'aide des différents indices calculés, la diversification des exportations ivoiriennes se présentent à travers l'évolution de l'indice de Theil comme suit :

Figure 7: Indice de THEIL Global

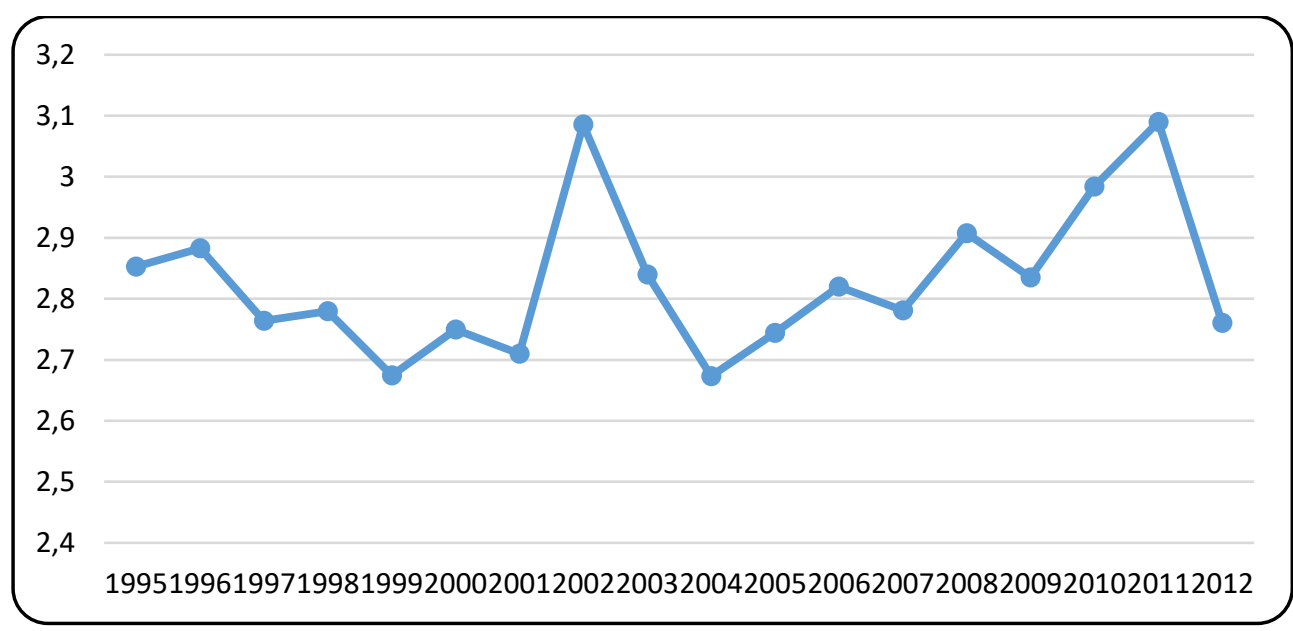

Source : Auteur, à partir des données sur Unctadstat, 2013

L'analyse du graphique nous montre que de 1995 à 2001, l'indice est en baisse. Il a connu une hausse de 2001 à 2002. A partir de cette date, il a baissé jusqu'en 2004. La diversification des exportations a repris jusqu'à 2010. Cette évolution peut s'expliquer par le rôle joué par l'APEX-CI, comme appui 
institutionnel auprès des entreprises exportations ivoiriennes. La baisse des activités de diversification des exportations s'explique par les crises socio-politiques connues en 2002 et 2010. Globalement, les exportations ivoiriennes sont très moyennement diversifiées (2\%<indice de Theil<.10\%).

La décomposition de l'indice de Theil nous permettra de savoir si les exportations ivoiriennes sont faiblement, très moyennement ou fortement diversifiées.

\section{Indice de Theil décomposé}

L'évolution des exportations ivoiriennes à l'aide du calcul des marges intensives et extensives se présente comme suit :

Figure 8:Indice décomposé de Theil

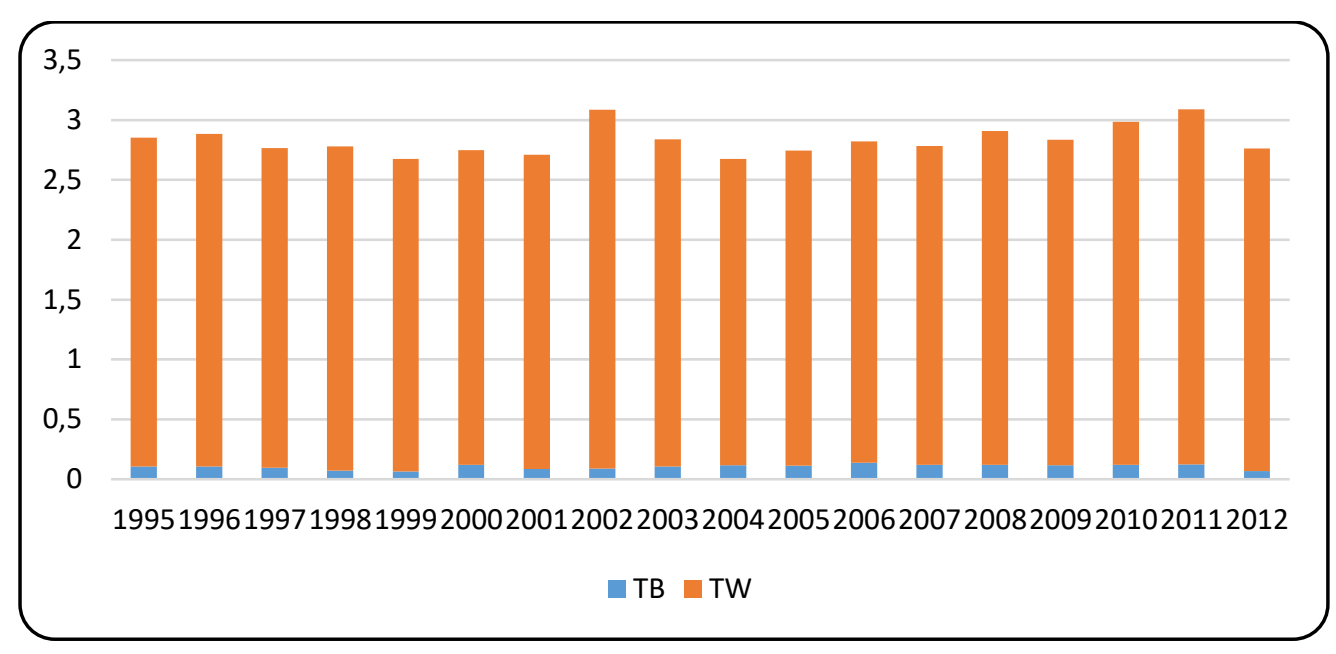

Source : Unctad, 2013

L'analyse des marges indiquent que les marges extensives (TB) sont très faibles par rapport aux marges intensives (TW). Ces nouveaux outils nous renseignent que les exportations ivoiriennes souffrent de la diversification des lignes de produits (très faibles marges extensives). Néanmoins, les exportations ivoiriennes sont maintenues et croissent $(2 \%<$ marges intensives< $10 \%)$. Cependant, la durabilité des exportations sera fonction des évolutions des prix des matières premières sur les marchés mondiaux. Elle est donc indépendante de la politique commerciale.

La politique de diversification en Côte d'Ivoire n'a pas encore contribué à favoriser intensément les exportations ivoiriennes. Toutefois, elle a contribué à accroitre ses exportations (les marges intensives sont situées au-delà de $2 \%$, mais n'atteignent pas $10 \%$ ). La politique de diversification horizontale qui vise de nouveaux secteurs d'activités peut possiblement être atteinte si l'Etat favorise l'exploitation des ressources dont dispose la Côte d'Ivoire. Pour ce qui concerne la politique de diversification verticale, le coût de la technologie et l'absence de sophistication des produits constituent un handicap pour la Côte d'Ivoire, contrairement aux autres pays en développement comme l'Inde et la Chine qui 
ont remarquablement bien réussi à diversifier leur économie et filières d'exportation. Au moins, $80 \%$ des exportations sont des produits industriels, contre $20 \%$ en Afrique subsaharienne où seulement $20 \%$, mais faiblement transformés (Gelb, 2010).

\section{Conclusion}

La diversification économique et plus spécifiquement la diversification des exportations commerciales internationale est au cœur des débats économiques actuels.

Le libéralisme économique a permis à la Côte d'Ivoire de s'inscrite dans une politique commerciale de diversification internationale de ses exportations en favorisant la création d'une institution de promotion des exportations.

L'objectif de notre étude est d'analyser le degré de diversification des exportations ivoiriennes à travers des instruments de mesures autres que les indicateurs traditionnels comme l'indice de GINI. A cause des avantages comparatifs et des gains issus des échanges en vertu des théories du commerce international, les exportations sont longtemps dominées par les produits essentiellement agricoles. La structure des exportations est dominée par les matières premières et quelques produits manufacturés. En dépit de la volonté du gouvernement ivoirien d'industrialiser sa production, le tissu industriel reste faible. Son ouverture au commerce international est un moteur de sa croissance économique. Mais, la transformation structurelle de l'économie a encore du chemin à parcourir.

Néanmoins, le pays s'est doté d'une Association de promotion des exportations (APEX CI). Toutefois, malgré les progrès importants de l'institution en matière de promotion des exportations et de visibilité (la Côte d'Ivoire est membre de l'AGOA${ }^{1}$ ), les résultats restent mitigés. En effet, les exportations du pays sont croissantes et la balance commerciale est excédentaire (Unctadstat, 2014). Cependant l'analyse profonde de la structure des exportations à travers l'indice de Theil montre que la marge intensive est certes relativement élevé, signe d'un accroissement des exportations mondiales, mais la marge extensive est très faible ; ce qui veut les exportations ivoiriennes sont très peu diversifiées. Ces caractéristiques traduisent le fait qu'il y a un blocage au niveau de la création de nouvelles lignes de produits, voire même au niveau de la sophistication des échanges.

\footnotetext{
${ }^{1}$ L'AGOA, est une loi américaine créée depuis 2000 qui vise à renforcer les relations économiques entre les Etats-Unis (USA) et l'Afrique.

Elle a pour objet de promouvoir les exportations vers les USA par des mécanismes de renforcement de capacités, d'investissements et de financements.

Cette loi contient des dispositions générales qui stipulent que les pays africains peuvent exporter leurs produits sur le marché américain sans droits de douanes et sans quota, et ce, jusqu'en 2015.
} 
Ce résultat est identique aux études menées sur les pays africains de la zone franc (CEA, 2012) et ceux de l'Afrique centrale (Banque de France, 2007).

Ce résultat implique pour nous de faire des suggestions à l'endroit des acteurs du commerce international en passant par les décideurs publics.

D’après nos résultats, la marge intensive des exportations est moyennement élevée.

Comme les exportations de la Côte d'Ivoire sont dominées par les produits agricoles comme le cacao, le coton, et le caoutchouc, elle doit encadrer les producteurs de sorte à ce que ces derniers continuent de produire davantage pour conserver sa place de leader dans la production cacaoyère par exemple. Pour les autres produits agricoles, elle doit chercher à améliorer sa production :

Comme la marge extensive est faible, la Côte d'Ivoire doit mettre l'accent sur une politique $d^{\prime}$ industrialisation à travers la promotion des investissements directs étrangers que mène déjà le CEPICI (centre de promotion des investissements). L'Etat de Côte d'Ivoire pourrait ainsi faciliter l'exploitation, la transformation et les exportations des ressources naturelles encore en l'état ;

Compte tenu du rôle de l'appui institutionnel de l'Apex- ci, qui jouit de la confiance de l'OMC et des bailleurs de fonds, son rôle mérite d'être renforcé, afin d'assister davantage les entreprises ivoiriennes qui exportent les produits manufacturés et celles qui voudraient s'engager à exporter des produits sophistiqués. La sophistication est aussi un déterminant de la diversification internationale pour les pays africains qui souhaitent davantage exportés plusieurs gammes de produits.

La Côte d'Ivoire doit tout faire pour bénéficier de son degré d'ouverture qui se situe à au moins $80 \%$ (Unctadstat, 2014).

\section{BIBLIOGRAPHIE}

[1] Azam J.-P., Ch. Morrisson (1994), La faisabilité politique de l'ajustement en Côte d'Ivoire et au Maroc. Paris, Centre de Développement, OCDE, $129 \mathrm{Pp}$.

[2] BANQUE DE FRANCE (2007), Diversification économique en Afrique centrale : état des lieux et enseignements ", Rapport zone franc, pp. 113-129, Paris.

[3] Ben Hammouda H., Karingi S.N., Njuguna A. et Sadni-Jallab M. (2006), " La diversification, vers un nouveau paradigme pour le développement de l'Afrique", Document de travail ATPC, Commission Economique pour l'Afrique, Nations Unies.

[4] Berezin P., Salehizadeh A., Santana E. (2002), "The challenge of diversification in the Caribbean", FMI, Working Paper $n^{\circ}$ 02/196.

[5] Berthélemy Jean Claude (2005), "Commerce international et diversification international" Revue d'économie politique, $\mathrm{Pp}$; 595-611.

[6] Bertinelli, L., V. Salins, and E. Strobl. (2006) "Export diversification and price uncertainty in SubSaharan Africa and other developing countries: a portfolio theory approach", mimeo, Ecole Polytechnique, Paris X University.

[7] Besedes T. et T.J. Prusa (2011) "The Role of Extensive and Intensive Margins and Export Growth", Journal of Development Economics, Elsevier, 96(2), pp. 371-379. 
[8] Cadot, O., C. Carrère et V. Strauss-Kahn (2011) "Export diversification: What's behind the hump?", The Review of Economics and Statistics, 93(2): 590-605.

[9] Cadot O., Carrère C. et Strauss-Khan V. (2009), "Export diversification, what's behind the hump?", Clermont-Ferrand, Document de travail, Université d'Auvergne CERDI.

[10] Cadot, O., A. Fernandes, J. Gourdon, and A. Mattoo (2012). "Are the benefits of export support durable? evidence from Tunisia". Technical report, The World Bank. Policy Research Working Paper, n. 6295.

[11] CAPEC et PNUD (2014), Les moteurs de la croissance et l'ouverture commerciale en Côte d'Ivoire, Rapport d'études.

[12] CEA (2013), Diversification et sophistication comme levier de transformation structurelles des économies Nord-africaines, document de la commission économique pour l'Afrique, publication de la commission économique pour l'Afrique.

[13] Contamin B. (1999), La libéralisation douanière de la Côte d'Ivoire : une politique sous conditions, in Gastellu J-.M., J.-Y. Moisseron, G. Pourcet (éds), Fiscalité, Développement, et Mondialisation, Maisonneuve \& Larose, DIAL, ORSTOM.

[14] Cottet C., Madariaga N., Jegou N. (2012), "la diversification en zone franc : degré, Sophistication et dynamique", Macroéconomie et développement, №3.

[15] Cruz Marcio (2014), "Do export promotion agencies promote new exporters" Interamerican bank, integration and trade sector.

[16] Danik Rodrik (2002), "Politiques de diversification" Revista Cepal, Numéro H.S, Pp 31-48.

[17] Dogruel, S. et M. Teckce (2011) "Trade liberalization and export diversification in selected MENA countries", Topics in Middle Eastern and African Economies, №13, Pp 1-24.

[18] Gaskani R. , Mehdi T. , M. B Mistry (2011), "Export instability and Economic growth (the case of OPEC)", International journal of finance, accounting and economic studies, Vol.1, N ${ }^{\circ} 2$, pp. 39-55.

[19] Gelb A. (2010) "Diversification de l'économie des pays riches en ressources naturelles", Document de Travail, Center for Global Development, Washington DC.

[20] Gourdon, J. (2010) " FDI flows and export diversification: looking at extensive and intensive margins", in: Lopez-Calix, P. Walkenhorst et N. Diop eds. Trade Competitiveness of the Middle East and North Africa, The World Bank.

[21] Hausmann, Ricardo, Bailey Klinger and Jose Lopez-Calix (2010) "Export Diversification in Algeria" in Lopez-Calix, Jose, Peter Walkenhorst and Ndiame Diop (eds) Trade Competitiveness of the Middle East and North Africa, World Bank, Washington DC.

[22] Hayakawa K., Lee H.H. Park D. (2011), "Do export promotion agencies increase exports?" Institute of developing economies, discussion paper, Pp. 313.

[23] Hesse, H. (2009) "Export diversification and economic growth", in: Breaking into new markets: Emerging lessons for export diversification, Washington: World Bank, pp.55-80.

[24] Imbs J. et Wacziarg R. (2003), "Stages of diversification", American Economic Review, vol. 93 (1), pp. 63-86.

[25] ITC (2014), Le commerce pour le bien de Tous, Rapport d'études.

[26] Jacquemin, A. P. and C.H. Berry "Entropy measure of diversification and corporate growth", The journal of industrial economics, 27(4), pp. 359-369.

[27] Klinger, B. et D. Lederman (2011), "Export discoveries, diversification and barriers to entry", Economics Systems, 35: 64-83

[28] Klinger B. et D. Lederman (2006), "Diversification, Innovation, and Imitation Inside the Technological Frontier", Research Policy Working Paper No 3872, Banque Mondale, Washington DC.

[29] Kubrak Claire (2013), Concentration et spécialisation des activités économiques : des outils pour analyser les tissus productifs locaux, Document de travail, INSEE

[30] Lederman d. et W.F. Maloney (2007), "Natural Resources: Neither Curse nor Destiny", Banque Mondiale et Stanford University Press, Washington DC. 
[31] Levchenko, A., and J. Di Giovanni. (2008), The risk content of exports: a portfolio view of international trade. Mimeo. The International Monetary Fund.

[32] Lim, J. et C. Saborowski (2012) "Export diversification in a transitioning economy ", Economics of Transition, 20(2), Pp. 339-367.

[33] Massell B. F. (1970), "Export Instability and Economic Structure", American Economic Review, vol. $60(4)$, p. $618-630$.

[34] Melitz, M. (2003), "The impact of trade on intra-industry reallocations and aggregate industry productivity", Econometrica, 71(6): 1695-1725

[35] Nicet-Chenaf Dalila et Rougier Eric (2008), "Recent exports matter: export discoveries, FDI and growth, an empirical assessment for MENA countries", GRETHA

[36] OECDE, NEPAD (2011), Economic diversification in Africa, A review of selected countries, Rapport d'études.

[37] OMC (1995, 2012), Examen des politiques commerciales sur la Côte d'Ivoire, Rapport du Secrétariat Général

[38] Prebisch, Raul (1950), "The Economic Development of Latin America and its Principal Problems". Economic Bulletin for Latin America 7: 11-22.

[39] Romer P. (1990), "Endogenous Technological Change", Journal of Political Economy, vol. 98, p. 71102.

[40] Theil, H. (1972), "Statistical Decomposition Analysis"; North Holland.

[41] UNCTAD (2014), profil général de la Côte d'Ivoire. 